\title{
ANÁLISE COMPARATIVA DOS RESULTADOS OBTIDOS PELOS MÉTODOS DE CALIBRAÇÃO DE CAMPO TRIDIMENSIONAL E BIDIMENSIONAL
}

\author{
Comparative analysis of results obtained by calibration with three-dimensional \\ and two-dimensional field methods
}

MARIANA BATISTA CAMPOS ${ }^{1}$

ANTONIO MARIA GARCIA TOMMASELLI ${ }^{1}$

MARCUS VINICIUS ANTUNES DE MORAES ${ }^{1}$

JOSÉ MARCATO JUNIOR ${ }^{2}$

1Universidade Estadual Paulista Júlio de Mesquita Filho, FCT/UNESP, Programa de Pós Graduação em Ciências Cartográficas

Email: marianaa.bcampos@gmail.com; tomaseli@fct.unesp.br; irmarcato@gmail.com

2 Universidade Federal de Mato Grosso do Sul, FAENG,UFMS, Campo Grande.

Email :antunesdemoraes@gmail.com

\section{Resumo:}

Os recursos de automação têm facilitado a utilização da Fotogrametria Digital em várias aplicações. Um processo complexo e crítico em Fotogrametria, a calibração de câmaras, pode ser realizado automaticamente, existindo programas comerciais com essa capacidade. Dentre os métodos de calibração, destaca-se o método de calibração de campo, usando conjunto de pontos bidimensionais ou tridimensionais. Alguns programas comerciais utilizam um modelo de campo bidimensional (Placa 2D) que possibilita o reconhecimento automático de alvos, como por exemplo, o PhotoModeler. Entretanto, o campo de calibração tridimensional pode ser mais adequado para algumas aplicações, como por exemplo, para a modelagem tridimensional de objetos. No intuito de avaliar a influência nos parâmetros de orientação interior decorrente do uso de campos 2D ou 3D, foram realizadas 3 calibrações, utilizando 2 campos $2 \mathrm{D}$ e 1 campo $3 \mathrm{D}$, as quais foram processadas em programas comercial e científico (PhotoModeler e Calibração com Múltiplas Câmaras). A forma de análise dos resultados consistiu em uma análise comparativa entre as calibrações, pelo estudo de influência dos Parâmetros de Orientação Interior (POI) na reconstrução de coordenadas de alvos sinalizados em um tripleto de imagens. Foram simuladas duas situações distintas (orientação exterior direta e indireta) e por fim, verificou-se o impacto dos POI nas coordenadas no plano e fora deste plano. A calibração com campo 3D, se comparada à calibração com o campo 2D, permitiu redução de correlação entre a distância focal e a coordenada $\mathrm{Z}$ do centro perspectivo $\left(\mathrm{Z}_{0}\right)$. Verificou-se esse resultado na orientação direta e indireta. Observou-se, também, que o método de calibração 3D 
leva a melhores resultados no cálculo das coordenadas do tripleto de imagens para os alvos fora do plano.

Palavras-chave: Calibração de Câmaras; Método de Calibração de Campo; Correlação de Parâmetros; Photomodeler.

\begin{abstract}
:
Automation resources have been used in Digital Photogrammetry in various applications. A complex and critical process in Photogrammetry, the camera calibration, can be performed automatically. Among the methods for camera calibration, field calibration using a set of twodimensional or three-dimensional points can be mentioned. Some commercial software uses a 2D plotted sheet, which allows the automatic recognition of targets, such as Photomodeler. However, the three-dimensional calibration field can be more suitable for certain applications, for example, three-dimensional object modeling. Thus, it is relevant to assess the effects of the interior orientation parameters, resulting from the use of these techniques, in particularly to correlation between parameters. For this purpose, three calibrations trials were performed using three different test fields (two 2D fields and one 3D field), processed in commercial and scientific software's (Photomodeler and Calibração com Múltiplas Câmaras). The results were analyzed by comparing calibrations, assessing the influence of the interior orientation parameters in the reconstruction of targets coordinate in an image triplet. Two different situations (direct and indirect exterior orientation) were simulated and it was verified the impact of the interior orientation parameters in the in-plane and out-of-plane coordinates. The calibration with 3D field, compared to 2D field, reduced the correlation between the focal length and the $\mathrm{Z}$ coordinate of the perspective center, showing better results for the correlation between parameters in both orientations (direct and indirect). It was also observed better results when using 3D calibration field for the calculation of out-of-plan targets coordinates.
\end{abstract}

Keywords: Camera Calibration; Field Calibration Method; Parameters Correlation; Photomodeler.

\title{
1. Introdução
}

O processo de calibração de câmaras é fundamental em Fotogrametria. A determinação dos parâmetros de orientação interior (POI) de cada câmara e sua confiabilidade, objetivo da calibração, possibilita a correção dos erros sistemáticos das medidas no espaço imagem, que afetam a precisão dos processos fotogramétricos posteriores.

Os POI ou parâmetros intrínsecos definem as características internas de uma câmara e permitem reconstruir analiticamente o feixe de raios formador da imagem. O conjunto de parâmetros mais empregado consiste em: distância focal, coordenadas do ponto principal, coeficientes de distorção radial simétrica, distorção descentrada e afinidade (Brown, 1971; Moniwa, 1972; Habib e Morgan, 2003).

A acurácia na determinação dos POI influencia nos produtos fotogramétricos como ortoimagens, Modelos Digitais de Terreno (MDT), modelos paramétricos de objetos, dentre outros (Galo et al., 2008).

Durante muitos anos as pesquisas relacionadas à calibração de câmaras foram voltadas para o aperfeiçoamento dos equipamentos, sendo que, em 1940, com o interesse militar durante a segunda guerra mundial, aumentaram os investimentos em equipamentos para calibração, como 
os Multicolimadores e Goniômetros, objetivando maior precisão e fácil manuseio, (Clarke e Fryer, 1998; Boland, 2000; Cramer, 2004; Merchant, 2012).

Grandes avanços nos modelos e métodos de calibração de câmaras ocorreram entre 1950 e 1970, destacando-se, além dos métodos de laboratório, os métodos de calibração de campo e o desenvolvimento do modelo de Conrady-Brown (Conrady, 1919; Eisenhart, 1963; Brown, 1966; Brown, 1971). Os diversos estudos relacionados à calibração de campo permitiram o desenvolvimento de variantes desse método, como por exemplo, o método de calibração plumbline (Brown, 1971), o método dos campos mistos (Merchant, 1979; Andrade e Olivas, 1981), autocalibração (Ebner, 1976; Brown, 1989; Fryer, 1996), câmaras convergentes (Kenefick et al, 1974), métodos de campo Tridimensional e Bidimensional (Merchant, 1979), dentre outros.

Com o desenvolvimento da Fotogrametria Digital e a automação de alguns processos, novas possibilidades de calibração facilitaram sua aplicação, como a identificação de alvos e medição das coordenadas no espaço imagem automaticamente.

Um avanço recente foi o desenvolvimento de programas comerciais, como por exemplo, o Photomodeler (Eos Systems inc, 2013), que, dentre outras funcionalidades, proporciona a calibração automática de câmaras a partir de alvos codificados pré-definidos. Este programa utiliza um padrão bidimensional para a calibração, que consiste de um conjunto de 4 alvos codificados e vários alvos circulares. Outros programas, inclusive em código aberto, também utilizam padrões bidimensionais (Heikkilä, 2000). Contudo, ressaltam-se as limitações desse tipo de configuração planar, sendo que os conjuntos tridimensionais produzem resultados mais robustos (Triggs, 1998; Zhang, 2000; Côrtes, 2010, Fraser, 2012).

Considerando esses avanços, a análise dos métodos de calibração em campo torna-se relevante diante da diversidade de câmaras e lentes, possibilitando verificar os benefícios das técnicas empregadas e suas limitações, principalmente no que concerne à correlação entre os parâmetros e a influência dos mesmos.

\section{Calibração de Câmaras}

A determinação dos parâmetros de orientação interior é realizada no processo de calibração (Andrade, 1998), possibilitando a análise do desempenho e da estabilidade da lente (Fryer, 1996).

Os métodos de campo permitem que todos os parâmetros sejam recuperados simultaneamente no processo de calibração, sendo requisito a superabundância de observações, o que torna possível um controle estatístico rigoroso no processo (Andrade, 1998).

Em geral, o processo de calibração de câmaras em campo pode ser realizado através de algumas etapas: (1) determinação de pontos com coordenadas conhecidas no espaço objeto (pontos de apoio), por meio de técnicas topográficas ou usando a tecnologia GNSS (Global Navigation Satellite System), por exemplo; (2) extração de coordenadas no espaço imagem, com acurácia da ordem do pixel ou subpixel, o que depende do tipo de alvo implantado no campo e do aplicativo usado para medição das coordenadas; (3) processamento da calibração com ajuste por feixes de raios e; (4) análise estatística para avaliara significância dos parâmetros, considerando a influencia de cada um deles no erro total modelado.

Os métodos de campo que utilizam conjunto de pontos $2 \mathrm{D}$ ou 3D, aliados ao método de câmaras convergentes, propiciam bons resultados com relação à redução de correlação entre os parâmetros de orientação interior e exterior. O método de câmaras convergentes consiste na tomada de fotografias convergentes, com no mínimo uma imagem, com rotação em torno do 
eixo z, com kappa variando entre $0^{\circ},-90^{\circ}, 90^{\circ}$ e $180^{\circ}$, visando solucionar os problemas de dependência linear entre alguns parâmetros, o que dificulta a determinação dos efeitos individuais de cada um (Andrade, 1998).

Apesar dos inúmeros estudos e métodos relacionados à calibração de câmaras, o desenvolvimento digital incentivou novas pesquisas no assunto, sendo um ponto de discussão na comunidade científica, enfatizando-se a importância da calibração para aumentar a exatidão dos processos (Mitishita e Olivas, 2001; Telles e Tommaselli, 2005; Bazan et al, 2009; Debiasi et al, 2012; Mitishita et al, 2012). Uma tendência é o desenvolvimento de programas que propiciam a calibração automática de câmaras, direcionada principalmente ao usuário não especialista. Dentre eles, destaca-se o Photomodeler, que, além da calibração automática, possibilita realizar medidas e a modelagem 3D de objetos, baseado nas técnicas de Fotogrametria Terrestre (Eos Systems inc, 2013).

Uma das aplicações mais antigas da Fotogrametria Terrestre é destinada ao registro arquitetônico, sendo que a calibração utilizando campo bidimensional é amplamente utilizada, principalmente pela facilidade de implantação dos modelos de campo $2 \mathrm{D}$, com alvos de reconhecimento automático, disponibilizados por programas comerciais (Wang et al, 2008; Andrade e Schuler, 2011; Córdoba, 2012).

Além dos programas comerciais, existem os programas de calibração científicos, como por exemplo, o CMC (Calibração com Múltiplas Câmaras), desenvolvido pelo grupo de Pesquisa em Fotogrametria da Faculdade de Ciência e Tecnologia da Universidade Estadual Paulista (FCT/UNESP). O CMC foi desenvolvido em linguagem $\mathrm{C} / \mathrm{C}++$ e possui modelos de calibração para câmaras perspectivas convencionais e câmaras com lentes "olho de peixe", com estimação pelo Método dos Mínimos Quadrados, modelo combinado. Além disso, permite a calibração com injunções de estabilidade da orientação relativa (Ruy, 2008; Bazan et al., 2009).

Apesar das diferentes implementações, ambos os programas citados utilizam o modelo de correção de Conrady-Brown agregado às equações de colinearidade (Ruy, 2008; Bazan et al., 2009; Eos Systems inc, 2013).

\subsection{Modelo Funcional Básico}

O modelo de colinearidade baseia-se na condição geométrica de alinhamento entre um ponto no espaço objeto, seu respectivo ponto imagem e o centro perspectivo da câmara (Wolf e Dewitt, 2000).

Para realizar o processo de calibração, são adicionados às equações de colinearidade, os modelos paramétricos dos efeitos das distorções radial simétrica, descentrada e da afinidade. Assim as equações de colinearidade podem ser reescritas como nas Equações 1 e 2 (Fryer,1996):

$$
\begin{aligned}
& x=x_{0}-\Delta_{k_{x}}-\Delta_{p_{x}}-\Delta_{a_{x}}-f \cdot \frac{r_{11}\left(X-X_{0}\right)+r_{12}\left(Y-Y_{0}\right)+r_{13}\left(Z-Z_{0}\right)}{r_{31}\left(X-X_{0}\right)+r_{32}\left(Y-Y_{0}\right)+r_{33}\left(Z-Z_{0}\right)} \\
& y=y_{0}-\Delta_{k_{y}}-\Delta_{p_{y}}-\Delta_{a_{y}}-f \cdot \frac{r_{21}\left(X-X_{0}\right)+r_{22}\left(Y-Y_{0}\right)+r_{23}\left(Z-Z_{0}\right)}{r_{31}\left(X-X_{0}\right)+r_{32}\left(Y-Y_{0}\right)+r_{33}\left(Z-Z_{0}\right)}
\end{aligned}
$$

Em que:

$\mathrm{x}$, y são as coordenadas de um ponto no sistema de máquina, com origem no centro geométrico da imagem e paralelo ao sistema fotogramétrico;

$\mathrm{X}, \mathrm{Y}$ e Z são as coordenadas do ponto em um referencial cartesiano do espaço objeto; 
$\mathrm{X}_{0}, \mathrm{Y}_{0}$ e $\mathrm{Z}_{0}$ são as coordenadas do centro perspectivo (CP) no referencial cartesiano do espaço objeto;

f é a distância principal da câmara, ou constante da câmara;

$\mathrm{r}_{\mathrm{ij}}$ são os elementos da matriz de rotação em função das rotações $\omega, \varphi$ e $\kappa$;

$\mathrm{x}_{0}$ e $\mathrm{y}_{0}$ são as coordenadas do ponto principal;

$\Delta_{\mathrm{k}}$ são os deslocamentos causados pela distorção radial simétrica nas direções x e y;

$\Delta_{\mathrm{p}}$ são os deslocamentos causados pela distorção descentrada nas direções x e y;

$\Delta_{\mathrm{a}}$ são os deslocamentos causados pela afinidade entre as direções x e y.

\subsection{Modelo de Distorções de Conrady-Brown}

Conrady (1919) realizou um estudo sobre a distorção das lentes, sendo o primeiro a desenvolver um modelo para a distorção descentrada. A partir dos trabalhos de Conrady, Brown (1966) desenvolveu um modelo de correção, no qual as distorções são classificadas em distorção radial simétrica e distorção descentrada (Brown, 1971; Fryer, 1996), posteriormente denominado de Conrady-Brown.

A distorção radial simétrica é o deslocamento radial de um ponto na imagem de sua posição correta, ou seja, uma mudança no ângulo entre o raio de luz e o eixo óptico, causado pela refração sofrida pelo raio de luz ao atravessar o sistema óptico (Fryer, 1996; Wolf e Dewitt, 2000). As Equações 3 e 4 permitem o cálculo da distorção radial simétrica nas componentes $x$ e y.

$$
\begin{aligned}
\delta_{x_{r}} & =\left(x-x_{0}\right)\left(k_{1} r^{2}+k_{2} r^{4}+k_{3} r^{6}\right) \\
\delta_{y_{r}} & =\left(y-y_{0}\right)\left(k_{1} r^{2}+k_{2} r^{4}+k_{3} r^{6}\right)
\end{aligned}
$$

Em que:

x, y são as coordenadas de um ponto no sistema de máquina, paralelo ao fotogramétrico; $\delta \mathrm{x}_{\mathrm{r}}$ é o efeito da distorção radial simétrica na componente $\mathrm{x}$.

$\delta y_{\mathrm{r}}$ é o efeito da distorção radial simétrica na componente y.

r é o raio, calculado pela Equação 5.

$\mathrm{k}_{1}, \mathrm{k}_{2}$ e $\mathrm{k}_{3}$ são os parâmetros da distorção radial simétrica.

$$
r=\sqrt{\left(x-x_{0}\right)^{2}+\left(y-y_{0}\right)^{2}}
$$

A distorção descentrada, por sua vez, é causada pela impossibilidade de alinhamento entre os eixos ópticos das lentes que compõem o sistema de lentes, causando um deslocamento na posição de um ponto na imagem (Andrade, 1998). Os efeitos dessa distorção podem ser quantificados pelas Equações 6 e 7.

$$
\begin{aligned}
& \delta_{x_{d}}=p_{1}\left(r^{2}+2\left(x-x_{0}\right)^{2}\right)+2 p_{2}\left(x-x_{0}\right)\left(y-y_{0}\right) \\
& \delta_{y_{d}}=p_{2}\left(r^{2}+2\left(y-y_{0}\right)^{2}\right)+2 p_{1}\left(x-x_{0}\right)\left(y-y_{0}\right)
\end{aligned}
$$

Em que:

$\delta x_{d}$ é o efeito da distorção descentrada na componente $\mathrm{x}$.

$\delta y_{d}$ é o efeito da distorção descentrada na componente y.

Bol. Ciênc. Geod., sec. Artigos, Curitiba, v. 21, nº 2, p.308-328, abr-jun, 2015. 
$p_{1}$ e $p_{2}$ são os parâmetros da distorção descentrada.

\subsection{Modelos de afinidade}

A diferença de escala entre os eixos x e y do sistema de coordenadas e a não ortogonalidade entre os mesmos, pode ser modelada matematicamente através dos parâmetros de afinidade (Moniwa, 1972).

Existem diversos modelos que possibilitam esta parametrização dos efeitos, dentre eles destacam-se o modelo para câmaras analógicas não métricas, proposto por Moniwa (1972), também aplicado para câmaras digitais (Tommaselli e Tozzi, 1990), e o modelo desenvolvido por Habib e Morgan (2003), implementado no programa CMC (Equações 8 e 9).

$$
\begin{gathered}
\delta_{x_{a}}=A\left(x-x_{0}\right)+B\left(y-y_{0}\right) \\
\delta_{y_{a}}=A\left(y-y_{0}\right)
\end{gathered}
$$

Em que, $\delta x_{a}$ é o efeito da não ortogonalidade e da diferença de escala entre os eixos na componente $\mathrm{x} ; \delta y_{a}$ é o efeito da não ortogonalidade e da diferença de escala entre os eixos na componente y e $A, B$ são os parâmetros de afinidade.

\subsection{Coeficiente de Correlação}

Um dos problemas na calibração de câmaras é a correlação entre alguns parâmetros de orientação interior (POI) e orientação exterior (POE), principalmente quando são usados campos planos (2D). As principais correlações ocorrem entre os parâmetros: $x_{0}$ e $X_{0}, y_{0}$ e $Y_{0}, f \mathrm{e}$ $Z_{0}$, mas ocorrem também entre, $x_{0}$ e $\varphi$, e $y_{0}$ e $\omega$ (Andrade, 1998).

São várias as estratégias para reduzir a correlação entre os parâmetros, como por exemplo, os métodos de câmaras convergentes e campos mistos, a aplicação de injunções nos POE, dentre outros (Merchant, 1979; Andrade e Olivas, 1981; Andrade, 1998). Esta correlação pode ser quantificada através da matriz dos coeficientes de correlação. Os elementos da matriz dos coeficientes de correlação podem ser calculados pela Equação 10, a partir da matriz de covariância obtida no ajustamento pelo método dos mínimos quadrados (Galo et al, 2008).

$$
\rho_{i j}=\frac{\sigma_{i j}}{\sigma_{i} \sigma_{j}}
$$

Onde, $\sigma_{i}$ e $\sigma_{j}$ correspondem, respectivamente, aos desvios-padrão dos parâmetros i e j e $\sigma_{i j}$ é a covariância entre os parâmetros i ej.

\section{Campos de Calibração: Tridimensional e Bidimensional}


Para os estudos experimentais apresentados neste trabalho foram utilizados 3 campos de calibração, sendo um campo tridimensional e dois campos bidimensionais.

O campo de calibração 3D com alvos codificados foi instalado pelo grupo de pesquisa em Fotogrametria da FCT-UNESP, em 2013, no terraço do prédio da central de laboratórios do Departamento de Cartografia. É composto por 139 alvos (quadrados) codificados internamente no padrão ArUco (Silva et al., 2014; Aruco, 2012; Tommaselli, et al, 2013).Cada alvo propicia 4 medidas nos vértices do quadrado, numerados de 0 à 3 (Figura 1.a).

O processo de localização e identificação automática destes alvos é realizado no programa Localizador de alvos, que utiliza rotinas da biblioteca OpenCV (Silva et al., 2014), também desenvolvido pelo grupo de pesquisa e fornece coordenadas no espaço imagem com precisão subpixel.

As coordenadas de quatro pontos de apoio foram determinadas utilizando receptores GNSS, com rastreio de oito horas de duração e de forma simultânea. Foram ainda coletadas 42 imagens do campo de calibração utilizando uma câmara Hasselblad H4D de 50 Mpixels equipada com uma lente de $35 \mathrm{~mm}$, cedida pela empresa Sensormap (Moraes et al., 2013). Com os quatro pontos de apoio e as 42 imagens foi realizado o processo de fototriangulação para a determinação das coordenadas dos vértices dos demais alvos. A precisão obtida para as coordenadas destes pontos ficou em torno de $3 \mathrm{~mm}$.

Os campos bidimensionais utilizados consistem em duas placas planas, sendo que uma delas (Figura 1.b) utiliza o mesmo padrão de alvos ArUco que o campo 3D e a outra no padrão de alvos de reconhecimento automático do programa Photomodeler (Figura 1.c).

A placa 2D, com alvos no padrão ArUco, é composta por 48 alvos, totalizando 192 cantos que podem ser medidos. Os pontos de controle foram medidos utilizando os valores nominais da impressão, conferidos com medidas de distância realizadas com um paquímetro de 2 metros, com precisão de $0,2 \mathrm{~mm}$. A placa 2D, com alvos no padrão do PhotoModeler, possui 4 alvos codificados nos cantos que são identificados pelo código circular e 96 alvos circulares, identificados automaticamente por vizinhança, contabilizando 100 alvos. Para refinar a localização automática dos alvos, o PhotoModeler utiliza o método LSM (Least Square Matching), atingido precisão subpixel (Alsadik et al., 2013).

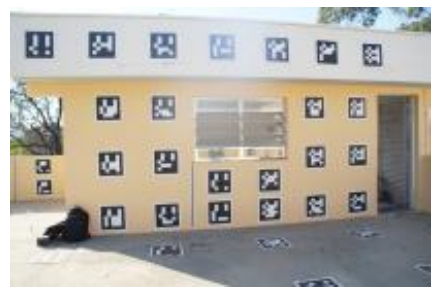

(a)

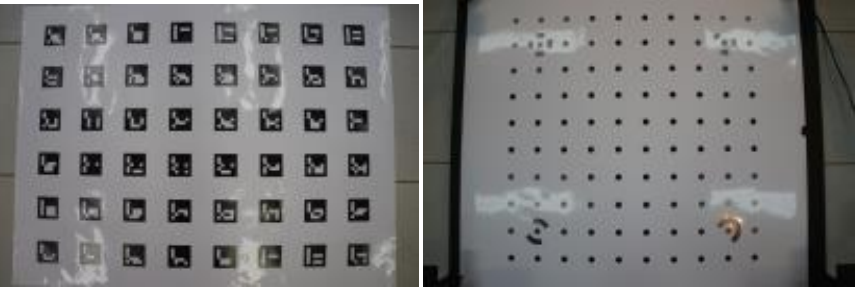

(b) (c)

Figura1: (a) Campo de Calibração 3D - FCT/UNESP, (b) Placa 2D no padrão ArUco, (c) Placa 2DPhotoModeler.

\section{Metodologia}


As técnicas aplicadas na aquisição e processamento dos dados visam atender a dois objetivos específicos: realizar uma análise comparativa entre os POI obtidos a partir dos campos de calibração $3 \mathrm{D}$ e $2 \mathrm{D}$ e avaliar a influência desses resultados em experimentos práticos de fototriangulação. Na aquisição das imagens para calibração utilizou-se a câmara Sony-Nex, com objetiva de $18 \mathrm{~mm}$. Os detalhes sobre a câmara utilizada são apresentados na Tabela 1.

Tabela 1: Informações técnicas sobre a câmara Sony Nex.

\begin{tabular}{c|c}
\hline Câmara & Sony Nex \\
\hline Distância Focal nominal & $18,0 \mathrm{~mm}$ \\
\hline Tipo/Tamanho do sensor & CMOS/23 mm x 15 mm \\
\hline Tamanho do pixel & $0,0047 \mathrm{~mm}$ \\
\hline Dimensões da imagem & $4912 \times 3264$ pixels \\
\hline
\end{tabular}

Para o processo de calibração e estimação dos POI, foram realizadas 3 coletas de dados, em três campos de calibração (Figura 1), baseadas no método de calibração em campo com ajustamento por feixes de raios e com a configuração de câmaras convergentes (Seção 3). Os três conjuntos de dados são caracterizados pelo campo utilizado (2D ou 3D), o programa de identificação automática dos alvos e o programa de processamento.

1) Calibração utilizando campo $3 \mathrm{D}$, com alvos no padrão ArUco, programa científico $\mathrm{CMC}$ e programa de identificação e medida automática de alvos (Localizador de alvos), ambos desenvolvidos pelo grupo de pesquisa em Fotogrametria da FCT/UNESP.

2) Calibração com placa $2 \mathrm{D}$, com alvos no padrão ArUco, programa científico CMC e programa de identificação e medida automática de alvos.

3) Calibração utilizando placa 2D, com alvos no padrão do PhotoModeler e o programa comercial PhotoModeler. A placa 2D de reconhecimento automático deste experimento é disponibilizada pelo próprio fabricante no site do programa.

A partir desses três conjuntos de dados foram processadas as calibrações obtendo-se os POI: distância focal, coordenadas do ponto principal, parâmetros da distorção radial simétrica e parâmetros da distorção descentrada. O programa CMC utiliza o modelo de afinidade apresentado por Habib e Morgan (2003). Entretanto os parâmetros de afinidade não foram utilizados na calibração da câmara, em nenhum dos experimentos porque o programa PhotoModeler, não possui modelo de afinidade, o que impossibilitaria a comparação entre os resultados das calibrações. Além disso, para câmaras digitais modernas o efeito da afinidade, na maioria dos casos é inferior ao erro de medida.

Em um primeiro momento, foi realizada uma análise individual da qualidade dos resultados de cada calibração, avaliando-se os desvios padrão dos POI, os resíduos das observações, o número de iterações e o sigma a posteriori, seguido de uma análise comparativa entre os valores obtidos nos campos 3D e 2D e por diferentes programas.

Considerando a importância dos POI no desenvolvimento dos produtos fotogramétricos, e com a crescente aplicabilidade da Fotogrametria na modelagem tridimensional de objetos, é importante avaliar a influência das diferenças entre os resultados dos valores de POI obtidos nos diferentes processos de calibração. Nesse sentido, avaliou-se esta influência no cálculo das coordenadas 3D de pontos no espaço objeto a partir de um tripleto de imagens, estimando-se as discrepâncias entre as coordenadas calculadas e as coordenadas de referência dos alvos, através da média, desvio padrão e a Raiz quadrada do Erro Médio Quadrático (REMQ) das discrepâncias encontradas. 
A REMQ é estimada pela raiz quadrada da somatória das discrepâncias ao quadrado, como apresenta a Equação11.

$$
\mathrm{REMQ}=\sqrt{\frac{1}{\mathrm{~N}} \cdot \sum_{\mathrm{i}=1}^{\mathrm{n}}(\text { Estimado }- \text { Referência })^{2}}
$$

Uma vez determinados os POI por calibração de campo, pode se utilizar a câmara em projetos fotogramétricos, desde que estes parâmetros sejam estáveis. Para o cálculo das coordenadas de pontos fotogramétricos no espaço objeto foram realizados dois grupos de experimentos, que correspondem às técnicas comumente usadas na modelagem tridimensional de objetos. No primeiro experimento admitiu-se a hipótese de que a orientação exterior (OE) era conhecida (orientação direta obtida por Sistemas de Orientação Direta, como sistemas integrando receptores GNSS e Unidade Inercial ou por Topografia Clássica), possibilitando analisar a compatibilidade entre os três modelos. Em um segundo momento, considerou-se os parâmetros de OE desconhecidos (orientação indireta), estimando-os no processo de Fototriangulação.

Além disso, foram avaliadas as discrepâncias obtidas no plano que contém os pontos de controle (in-plane) e fora do plano (out-of-plane), separadamente, para discutir a influência dos parâmetros de orientação interior na acurácia das coordenadas em profundidades distintas (Fraser, 2012). Para isso foram utilizadas as coordenadas estimadas no processo de Fototriangulação, com orientação exterior indireta, e POI fixos apenas do experimento 1 e do experimento 2, que utilizam os mesmos programas de identificação automática de alvos e de processamento da calibração, constituindo dois conjuntos de dados compatíveis. Mais detalhes sobre os experimentos serão apresentados na Seção 5.

\section{Experimentos e Resultados}

O primeiro conjunto de experimento refere-se à calibração da câmara Sony Nex com os três conjuntos de dados descritos na Seção 4.

As imagens do campo de calibração 3D foram coletadas em 5 de setembro de 2013. Para os campos bidimensionais, as aquisições ocorreram em 6 de setembro de 2013, respeitando a mesma geometria de aquisição e sem alterar a focalização do sistema de lentes.

\subsection{Parâmetros de Orientação Interior}

O CMC e o PhotoModeler possuem algumas diferenças na forma de apresentação do resultado final. É necessário, portanto, compatibilizar os dados para uma análise coerente dos POI. Com relação às coordenadas do ponto principal, o CMC apresenta o resultado com relação ao centro da imagem, enquanto o PhotoModeler utiliza como origem o canto superior esquerdo da mesma. Logo, para compatibilizar as medidas, aplicou-se uma translação na origem do PhotoModeler para o centro da imagem e, a partir da nova origem, determinou-se as coordenadas do ponto principal no mesmo sistema de referência do CMC.

Outra diferença está nos parâmetros de distorção radial simétrica e de distorção descentrada. $\mathrm{O}$ CMC fornece os coeficientes de distorção, enquanto o PhotoModeler disponibiliza coeficientes para a correção da distorção, que possuem o sinal inverso. A Tabela 2 apresenta os POI 
estimados, já compatibilizados para o padrão utilizado pelo CMC, e os respectivos desvios padrão fornecidos pelos programas a partir dos três conjuntos de dados (Seção 4).

Tabela 2: - Parâmetros de orientação interior obtidos a partir dos três experimentos.

\begin{tabular}{c|c|c|c}
\hline POI & $\begin{array}{c}\text { Experimento 1 } \\
\text { (3D e CMC) }\end{array}$ & $\begin{array}{c}\text { Experimento 2 } \\
\text { (2D e CMC) }\end{array}$ & $\begin{array}{c}\text { Experimento 3 } \\
\text { (2D e PhotoModeler) }\end{array}$ \\
\hline $\mathrm{f}(\mathrm{mm})$ & $18,1456 \pm 0,004$ & $18,1749 \pm 0,010$ & $18,8180 \pm 0,006$ \\
\hline $\mathrm{x}_{0}(\mathrm{~mm})$ & $-0,0637 \pm 0,002$ & $-0,1417 \pm 0,005$ & $-0,2155 \pm 0,002$ \\
\hline $\mathrm{y}_{0}(\mathrm{~mm})$ & $-0,0299 \pm 0,002$ & $0,0141 \pm 0,004$ & $0,0134 \pm 0,003$ \\
\hline $\mathrm{k}_{1}\left(\mathrm{~mm}^{-2}\right)$ & $-5,9992 \times 10^{-4} \pm 7,88 \times 10^{-6}$ & $-5,8816 \times 10 \pm 7,03 \times 10^{-6}$ & $-5,358 \times 10^{-4} \pm 2,50 \times 10^{-6}$ \\
\hline $\mathrm{k}_{2}\left(\mathrm{~mm}^{-4}\right)$ & $5,8899 \times 10^{-7} \pm 1,207 \times 10^{-7}$ & $5,8606 \times 10^{-7} \pm 1,219 \times 10^{-7}$ & $6,823 \times 10^{-7} \pm 1,7 \times 10^{-8}$ \\
\hline $\mathrm{k}_{3}\left(\mathrm{~mm}^{-6}\right)$ & $3,412 \times 10^{-9} \pm 5,64 \times 10^{-10}$ & $2,817 \times 10^{-9} \pm 6,29 \times 10^{-10}$ & $0,000 \pm 000$ \\
\hline $\mathrm{p}_{1}\left(\mathrm{~mm}^{-1}\right)$ & $-1,1843 \times 10^{-5} \pm 3,47 \times 10^{-6}$ & $-1,5256 \times 10^{-4} \pm 5,56 \times 10^{-6}$ & $-1,573 \times 10^{-4} \pm 1,4 \times 10^{-6}$ \\
\hline $\mathrm{p}_{2}\left(\mathrm{~mm}^{-1}\right)$ & $2,1938 \times 10^{-5} \pm 3,3371 \times 10^{-6}$ & $\begin{array}{c}8,82707 \times 10^{-6} \pm 5,0859 \times 10^{-} \\
6\end{array}$ & $3,244 \times 10^{-5} \pm 2,0 \times 10^{-6}$ \\
\hline
\end{tabular}

\subsection{Análise Comparativa}

Com os dados homogeneizados foi realizada uma análise comparativa entre os resultados obtidos com o campo 3D e o campo 2D, calculando-se as discrepâncias dos POI entre os experimentos 1 e 2 , assim como,entre os experimentos 1 e 3 . A Tabela 3 mostra as diferenças entre os valores encontrados nos experimentos 2 e 3 com relação ao experimento 1.

Tabela 3: Diferenças entre os POI obtidos nos experimentos.

\begin{tabular}{c|c|c}
\hline Parâmetros & Exp. 1 - Exp. 2 & Exp.1- Exp.3 \\
\hline $\mathrm{f}(\mathrm{mm})$ & $-0,0293$ & $-0,6724$ \\
\hline $\mathrm{x}_{0}(\mathrm{~mm})$ & 0,0780 & 0,1517 \\
\hline $\mathrm{y}_{0}(\mathrm{~mm})$ & $-0,0440$ & $-0,0433$ \\
\hline $\mathrm{k}_{1}\left(\mathrm{~mm}^{-2}\right)$ & $1,17 \times 10^{-5}$ & $-6,41 \times 10^{-5}$ \\
\hline $\mathrm{k}_{2}\left(\mathrm{~mm}^{-4}\right)$ & $2,92 \times 10^{-9}$ & $-9,33 \times 10^{-8}$ \\
\hline $\mathrm{k}_{3}\left(\mathrm{~mm}^{-6}\right)$ & $5,95 \times 10^{-10}$ & $3,41 \times 10^{-9}$ \\
\hline $\mathrm{p}_{1}\left(\mathrm{~mm}^{-1}\right)$ & $1,41 \times 10^{-4}$ & $1,45 \times 10^{-4}$ \\
\hline $\mathrm{p}_{2}\left(\mathrm{~mm}^{-1}\right)$ & $1,31 \times 10^{-5}$ & $-1,05 \times 10^{-5}$ \\
\hline \multicolumn{3}{|c}{}
\end{tabular}

As maiores diferenças são encontradas na distância focal e na coordenada $\mathrm{x}$ do ponto principal $\left(\mathrm{x}_{0}\right)$. Apesar das diferenças nas geometrias dos campos de calibração $3 \mathrm{D}$ e $2 \mathrm{D}$, os parâmetros de distorção radial simétrica obtidos nas três calibrações, assim como os parâmetros de distorção descentrada são semelhantes. As curvas de distorção radial simétrica podem ser visualizadas na Figura 2. 


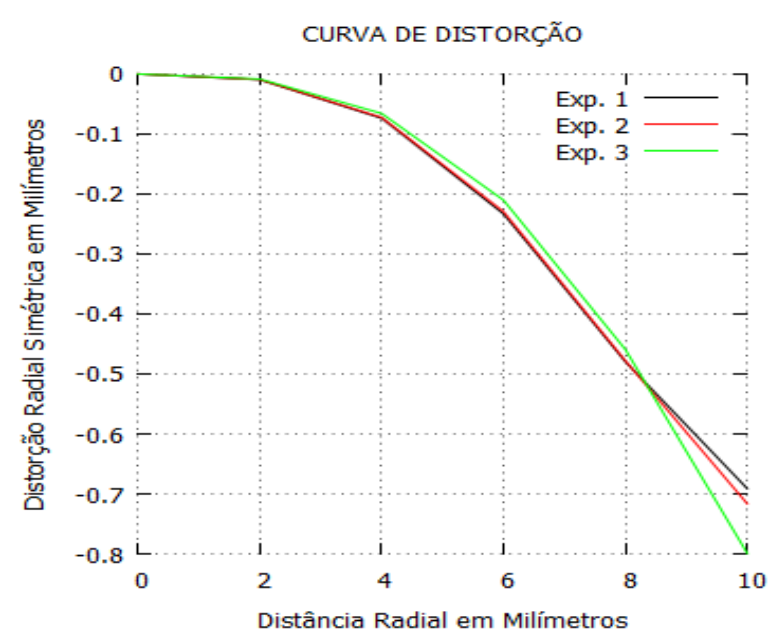

Figura 2: Curva da distorção radial simétrica.

Para avaliar a influência dos resultados obtidos e compreender o impacto das diferenças obtidas nos valores de $\mathrm{x}_{0}$, e principalmente, da distância focal, realizou-se um novo grupo de experimentos, nos quais objetiva-se calcular as coordenadas de terreno dos pontos de controle de um tripleto de imagens (Figura 3) e avaliar as discrepâncias encontradas.

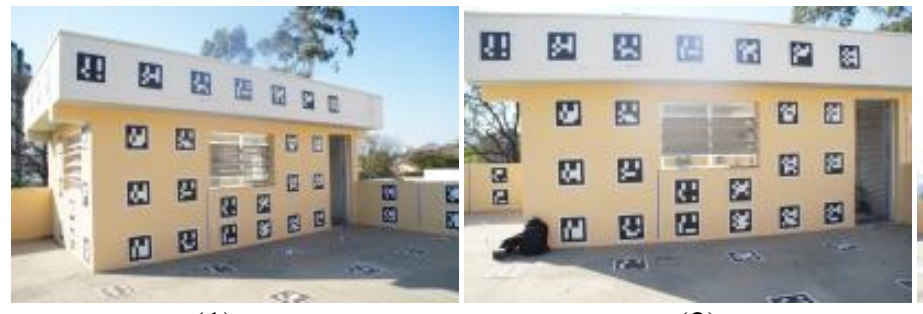

(1)
(2)

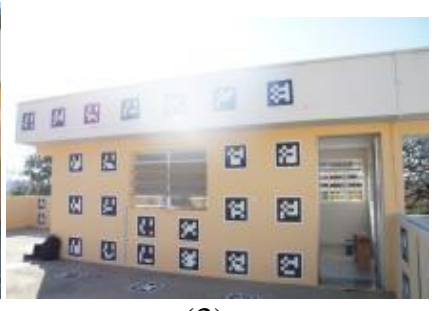

(3)

Figura 3: Tripleto experimental.

\subsection{Verificação da Influência dos POI com Orientação Exterior Direta}

As coordenadas no espaço objeto dos vértices dos alvos ilustrados na Figura 3 são conhecidas, determinadas por processos geodésicos e fotogramétricos, como citado na Seção 3. Para analisar a compatibilidade entre os modelos e os parâmetros obtidos, foram calculadas as coordenadas de terreno dos pontos, no programa CMC, fixando-se os POI obtidos em cada calibração e os parâmetros de orientação exterior (POE). Os POE utilizados neste experimento são provenientes da calibração 3D, que, por apresentarem boa precisão, podem ser considerados como referência, simulando um caso em que os POE fossem medidos diretamente. Os desvios padrão atribuídos às injunções foram: $5 \mathrm{~mm}$ e $0,005^{\circ}$ para os POE e nos POI, $0,5 \mu \mathrm{m}$ para a distância focal, $0,5 \mu \mathrm{m}$ em $\mathrm{x}_{0}$ e $\mathrm{y}_{0}, 1 \times 10^{-7} \mathrm{~mm}^{-2}, 1 \times 10^{-10} \mathrm{~mm}^{-4}, 1 \times 10^{-12} \mathrm{~mm}^{-6}$, para os coeficiente da distorção radial simétrica, $\mathrm{k}_{1}, \mathrm{k}_{2}$ e $\mathrm{k}_{3}$, respectivamente e $10^{-8} \mathrm{~mm}^{-1}$ para ambos os coeficientes de distorção descentrada ( $\mathrm{p}_{1}$ e $\left.\mathrm{p}_{2}\right)$.

Foram adotados apenas quatro pontos de controle bem distribuídos, com desvio padrão de 3 $\mathrm{mm}$, sendo o restante dos pontos considerados como pontos de verificação, totalizando 52 pontos de verificação. A Figura 4 ilustra a distribuição dos pontos de controle, destacados em 
vermelho. Neste caso, obtiveram-se as coordenadas de terreno dos pontos de verificação para os três conjuntos de parâmetros resultantes dos experimentos 1, 2 e 3.

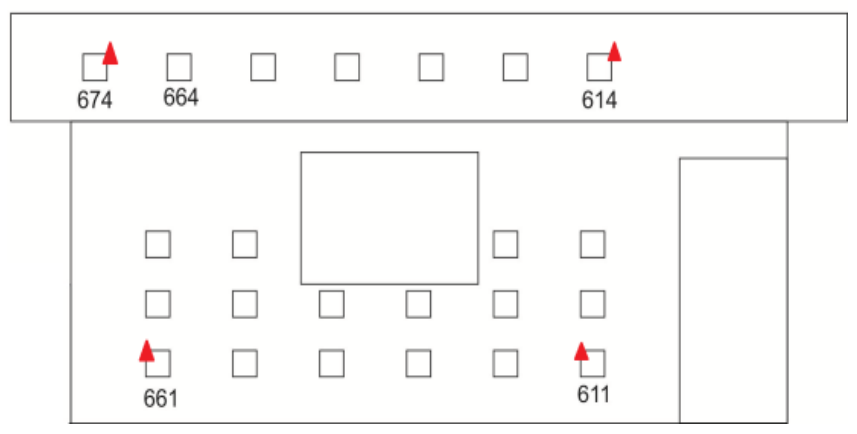

Figura 4: - Distribuição dos pontos de controle no tripleto.

Conhecendo-se as coordenadas de referência dos pontos de verificação, foram calculadas as discrepâncias entre as coordenadas de referência e as coordenadas calculadas por Fototriangulação. A média, desvio padrão e REMQ das discrepâncias são apresentados na Tabela 4.

Tabela 4: Média, desvio padrão e REMQ em pontos de verificação com o uso dos POI dos experimentos 1,2 e 3 e com POE conhecidos.

\begin{tabular}{c|c|c|c|c}
\hline \multirow{4}{*}{ Exp. 1 } & Estatísticas & $\mathbf{X}(\mathbf{m m})$ & $\mathbf{Y}(\mathbf{m m})$ & $\mathbf{Z}$ (mm) \\
\cline { 2 - 5 } & Média & $-0,727$ & 0,255 & 0,091 \\
\cline { 2 - 5 } & Desvio Padrão & 4,476 & 2,976 & 7,162 \\
\cline { 2 - 5 } & REMQ & 4,716 & 3,017 & 7,236 \\
\hline \multirow{3}{*}{ Exp. 2 } & Média & $-8,150$ & 3,923 & 0,048 \\
\cline { 2 - 5 } & Desvio Padrão & 7,512 & 4,393 & 7,748 \\
\cline { 2 - 5 } & REMQ & 11,256 & 5,976 & 7,826 \\
\hline \multirow{2}{*}{ Exp. 3 } & Média & $-21,750$ & 4,173 & 6,615 \\
\cline { 2 - 5 } & Desvio Padrão & 28,981 & 13,768 & 14,019 \\
\cline { 2 - 5 } & REMQ & 36,730 & 14,540 & 15,687 \\
\hline
\end{tabular}

Utilizando os POE fixos nota-se claramente, pela média das discrepâncias, a presença de tendência nos Exp. 2 e Exp. 3, sendo que o maior valor da REMQ está nas coordenadas X, que reflete as discrepâncias entre parâmetros $\mathrm{x}_{0}$,obtidos nos três experimentos (Tabela 3 ).

O Exp.1 reflete o resultado, esperado neste tipo de calibração, utilizando conjuntos de pontos tridimensionais, com REMQ das coordenadas $\mathrm{X}$ e $\mathrm{Y}$ próximas à precisão do campo utilizado $\mathrm{e}$ o maior valor da REMQ das discrepâncias na coordenada $Z$, em função da geometria da câmara. Observa-se, também, nos experimentos com campos bidimensionais, que o valor da REMQ na coordenada $\mathrm{Z}$ é superior ao obtido com o uso de campo tridimensional. Isso ocorre em função do valor da distância focal estimada em cada experimento. As discrepâncias obtidas em X, Y (a, b.c) assim como em XYZ (d,e,f), para cada ponto, ampliadas 50 vezes (50:1), podem ser visualizadas na Figura 5 na escala gráfica 1:200.

Este experimento mostra claramente os erros provocados pela fixação de parâmetros calculados em diferentes condições, como os calculados nos experimentos 2 e 3. Nota-se a presença de tendência nos experimentos 2 e 3 que utilizaram campos planos.

Apesar da relevância destes resultados, é importante lembrar que os POE utilizados nestes experimentos foram estimados durante o processo de calibração com o campo 3D, ou seja, os POI gerados no experimento 1 são compatíveis com estes POE, pois foram estimados 
simultaneamente. Para estimar independentemente os POE foram realizados os experimentos descritos na seção 5.4 .

(a)

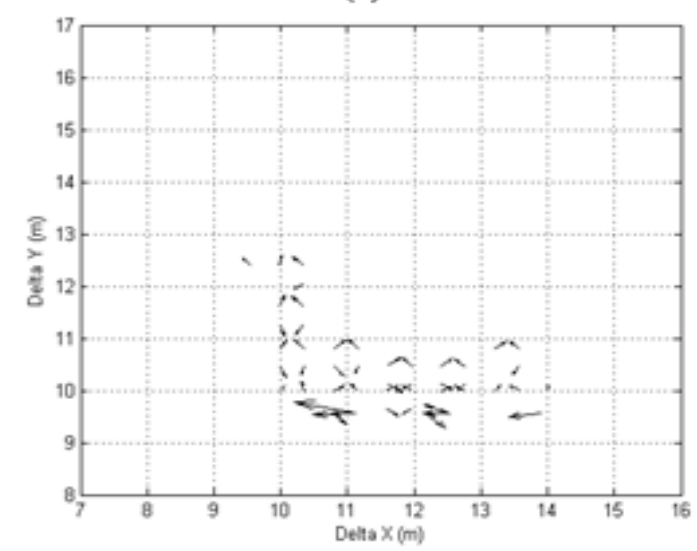

(b)

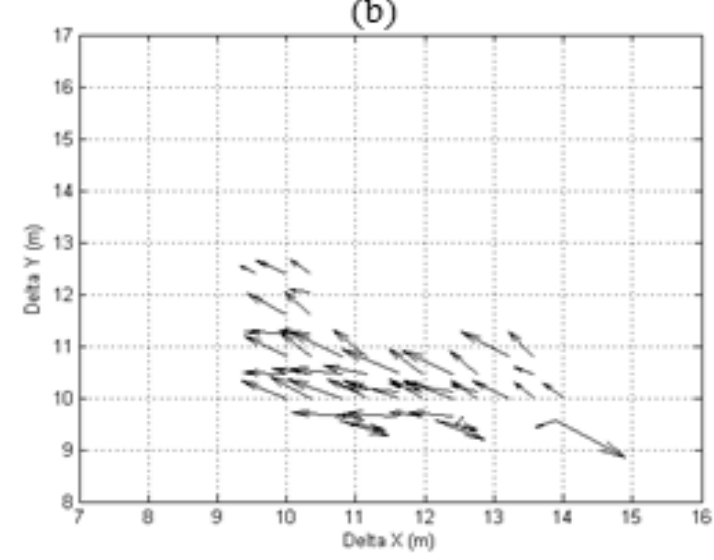

(c)

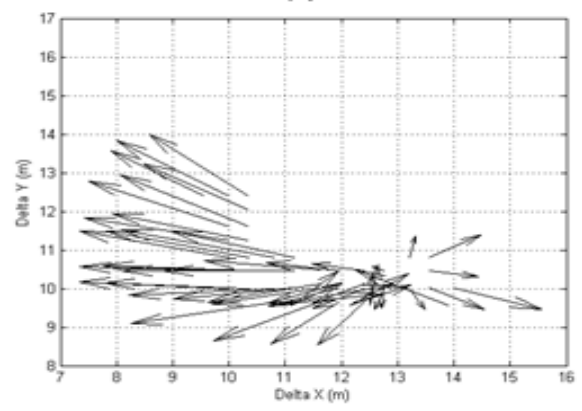

Escala Gráfica

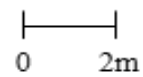

(d)

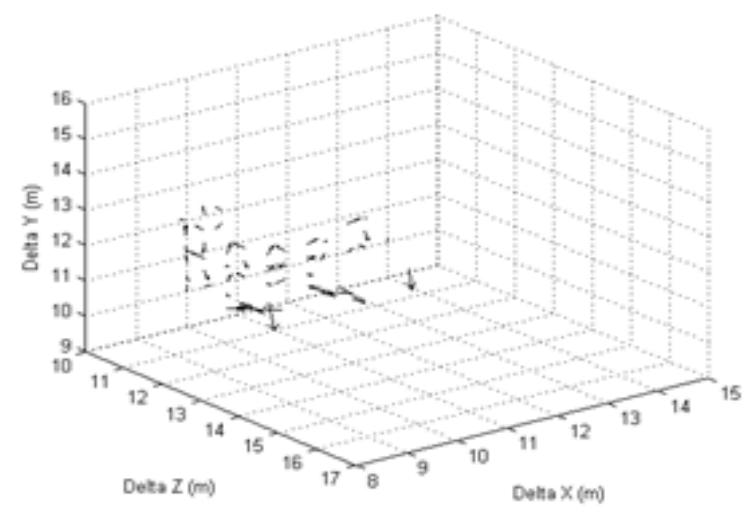

(e)

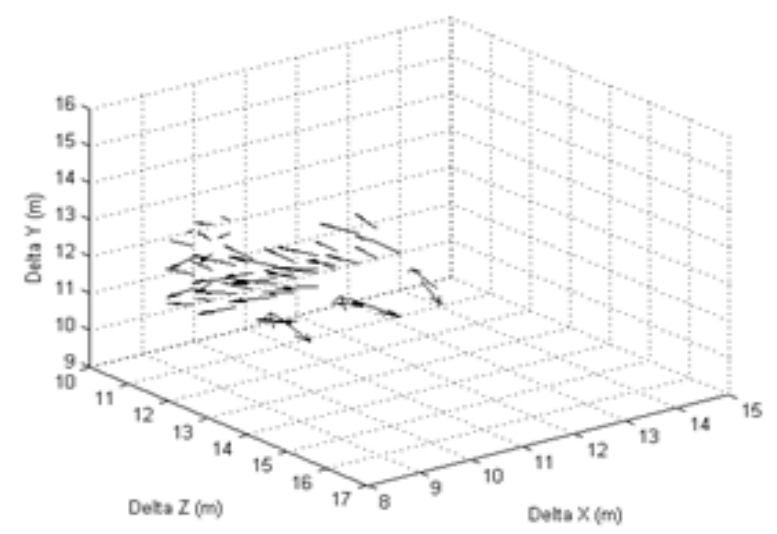

(f)

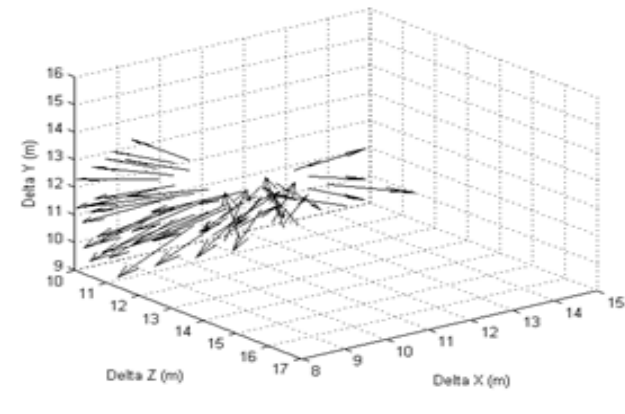

Escala dos residuos

$\stackrel{{ }_{0}}{\longrightarrow} 02 \mathrm{~m}$

Figura 5: Gráfico das discrepâncias planimétricas / planialtimétricas obtidas para cada ponto considerando em (a/d), (b/e), (c/f), o cálculo das coordenadas do modelo a partir dos POI obtidos nos experimentos 1, 2 e 3, respectivamente, com POE fixo. 


\subsection{Verificação da Influência dos Parâmetros de Orientação Interior, com a Determinação dos Parâmetros de Orientação Exterior Indireta}

A Fototriangulação é um processo fotogramétrico que objetiva determinar os parâmetros de orientação exterior e as coordenadas de terreno de pontos fotogramétricos. Nesse sentido, simulando essa aplicação, consideraram-se conhecidos apenas os POI,e tendo-se apenas os parâmetros aproximados para a orientação exterior, foi realizada uma Fototriangulação no programa CMC, sendo utilizados como apoio os quatro pontos apresentados na Figura 4, adotando também desvio padrão de $3 \mathrm{~mm}$

Foram fixados os POI obtidos em cada experimento de calibração para avaliar o impacto destes parâmetros na determinação da orientação exterior do tripleto e nas coordenadas no espaço objeto. Os desvios padrão atribuídos às injunções foram: $0,5 \mu \mathrm{m}$ para a distância focal, $0,5 \mu \mathrm{m}$ em $x_{0}$ e $y_{0}, 10^{-7} \mathrm{~mm}^{-2} \mathrm{em} \mathrm{k}_{1}, 10^{-10} \mathrm{~mm}^{-4} \mathrm{em} \mathrm{k}_{2}$, e $10^{-12} \mathrm{~mm}^{-6} \mathrm{em} \mathrm{k}_{3}$, e $10^{-8} \mathrm{~mm}^{-1}$ para ambos os coeficientes de distorção descentrada $\left(\mathrm{p}_{1} \mathrm{e} \mathrm{p}_{2}\right)$. Foram calculadas as discrepâncias entre as coordenadas conhecidas e as coordenadas obtidas em cada experimento de Fototriangulação e, posteriormente, a média, desvio padrão e REMQ das discrepâncias, apresentados na Tabela 5.

Tabela 5: Média, desvio padrão e REMQ referente à aplicação dos POI dos experimentos 1, 2 e 3.

\begin{tabular}{c|c|c|c|c}
\hline \multirow{4}{*}{ Exp. 1 } & Estatísticas & $\mathbf{X}(\mathbf{m m})$ & $\mathbf{Y}(\mathbf{m m})$ & $\mathbf{Z}(\mathbf{m m})$ \\
\cline { 2 - 5 } & Média & 0,351 & $-0,893$ & 0,780 \\
\cline { 2 - 5 } & Desvio Padrão & 4,063 & 1,310 & 5,503 \\
\cline { 2 - 5 } & REMQ & 4,079 & 1,296 & 5,606 \\
\hline \multirow{3}{*}{ Exp. 2 } & Média & 0,229 & $-3,250$ & 1,410 \\
\cline { 2 - 5 } & Desvio Padrão & 3,977 & 2,924 & 5,735 \\
\cline { 2 - 5 } & REMQ & 3,983 & 4,398 & 5,908 \\
\hline \multirow{3}{*}{ Exp. 3 } & Média & $-2,130$ & $-2,970$ & $-6,080$ \\
\cline { 2 - 5 } & Desvio Padrão & 9,100 & 9,096 & 6,409 \\
\cline { 2 - 5 } & REMQ & 9,350 & 9,579 & 8,879 \\
\hline
\end{tabular}

A média das discrepâncias permitiu detectar a presença de tendências que foram provocadas pelos POI utilizados e, portanto, por erros sistemáticos residuais, bem como pela geometria e distribuição dos pontos de controle. Nos experimentos 2 e 3,onde utilizou-se POI provenientes de calibrações com campos bidimensionais,observa-se uma maior tendência , principalmente, nas coordenadas Z. Entretanto é possível observar graficamente que as discrepâncias possuem padrão aleatório (Figura 6), ao contrário dos resultados apresentados na Figura 5, quando se utilizaram POE fixos.As discrepâncias obtidas em X, Y (a,b.c) assim como em XYZ (d,e,f),para cada ponto, ampliadas 50 vezes (50:1), podem ser visualizadas na Figura 6, com escala gráfica $1: 200$. 

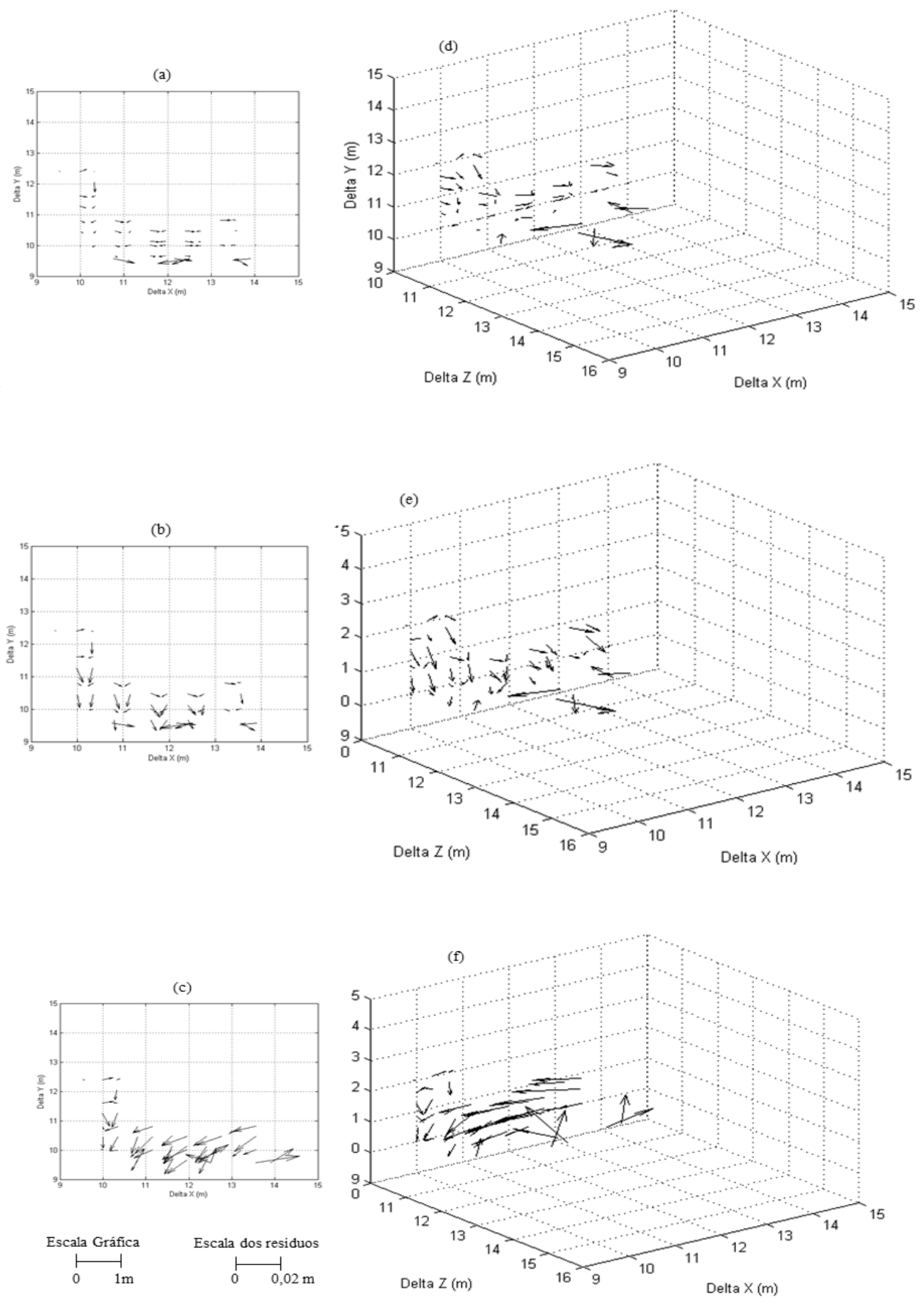

Figura 6: Gráfico das discrepâncias planimétricas / planialtimetricas obtidas para cada ponto considerando em (a/d), (b/e), (c/f), o cálculo das coordenadas do modelo a partir dos POI obtidos nos experimentos 1,2 e 3 , respectivamente, com POE estimados.

Neste caso, as discrepâncias entre os POI obtidos nos experimentos 2 (2D/CMC) e 3 (2D/PhotoModeler), com relação ao experimento 1 (3D/CMC), foram em grande parte absorvidas pelos POE, no processo de ajustamento, o que justifica a melhora dos resultados. Ainda assim, os melhores resultados foram aqueles obtidos com o campo tridimensional.

Os POE obtidos nas três calibrações apresentam discrepâncias de valores entre si, principalmente em função da absorção dos efeitos dos POI. Nesse sentindo, a Tabela 6 apresenta as discrepâncias entre os POE obtidos por Fototriangulação com POI estimados na calibração com o campo 3D e com POI estimados na calibração com ambos os campos 
bidimensionais. Na Tabela $6 \Delta \mathrm{E} 1-\mathrm{E} 2$ são as discrepâncias entre os POE obtidos com os campo $3 \mathrm{D}$ e2D, ambos com alvos no padrão ArUco e $\Delta \mathrm{E} 1-\mathrm{E} 3$ são as discrepâncias entre os POE relacionados aos POI obtidos com campo 3D, e aos POI do campo bidimensional no padrão PhotoModeler, ambos para as três imagens do tripleto.

Tabela 6: - Discrepâncias entre os POE calculados a partir dos POI obtidos na calibração com campos bidimensionais com relação ao campo tridimensional.

\begin{tabular}{c|c|c|c|c|c|c|c}
\hline & $\mathbf{I m a g e m}$ & $\left.\mathbf{\omega} \mathbf{(}^{\circ}\right)$ & $\boldsymbol{\varphi}\left(^{\circ}\right)$ & $\mathbf{K}\left(^{(}\right)$ & $\mathbf{X}_{\mathbf{0}}(\mathbf{m})$ & $\mathbf{Y}_{\mathbf{0}}(\mathbf{m})$ & $\mathbf{Z}_{\mathbf{0}}(\mathbf{m})$ \\
\cline { 2 - 8 } & 1 & 0,1271 & 0,2948 & $-0,0916$ & 0,0073 & 0,0028 & 0,0057 \\
\cline { 2 - 8 }$\Delta \mathbf{E 1 - E 2}$ & 2 & 0,1254 & 0,2220 & 0,0242 & $-0,0041$ & 0,0031 & 0,0087 \\
\cline { 2 - 8 } & 3 & 0,1720 & 0,2176 & 0,0965 & $-0,0085$ & 0,0005 & 0,0056 \\
\cline { 2 - 8 } & Média & 0,1415 & 0,2448 & 0,0097 & $-0,0017$ & 0,0021 & 0,0066 \\
\hline \multirow{3}{*}{$\Delta \mathbf{E 1 - E 3}$} & 1 & 0,6059 & 1,7422 & $-0,1559$ & 0,1564 & $-0,0478$ & 0,1062 \\
\cline { 2 - 8 } & 2 & 0,1376 & 0,6218 & 0,0495 & $-0,0255$ & 0,0113 & 0,1836 \\
\cline { 2 - 8 } & 3 & 0,3873 & 0,1809 & 0,0314 & $-0,1437$ & $-0,0239$ & 0,1430 \\
\cline { 2 - 8 } & Média & 0,3769 & 0,8483 & $-0,0249$ & $-0,0042$ & $-0,0201$ & 0,1442 \\
\hline
\end{tabular}

Em ambos os experimentos as maiores discrepâncias nos ângulos de atitude ocorreram no ângulo $(\varphi)$, que está correlacionado com a coordenada $X$, o que absorve as diferenças entre os valores de $\mathrm{x}_{0}$.

Quanto à posição, as maiores discrepâncias ocorreram em $\mathrm{Z}_{0}$, devido à correlação deste parâmetro com a distância focal. A maior discrepância em $Z_{0}$ é observada em $\Delta E 1-E 3$, devido à diferença significativa entre os valores da distância focal obtidos na calibração nos experimentos 1 (campo 3D) e 3 (POI estimados com o campo plano pelo Photomodeler), como apresentado na Tabela 5. Os coeficientes de correlação entre $f$ e $\mathrm{Z}_{0}$, e $\mathrm{x}_{0} \mathrm{e} \varphi$, para as três imagens do tripleto e para os três experimentos podem ser observados na Tabela 7.

Tabela 7: Coeficientes de correlação entre os POI e os POE nas três imagens do tripleto para os três experimentos.

\begin{tabular}{c|c|c|c|c}
\hline Parâmetros correlacionados & \multirow{2}{*}{ Experimentos } & \multicolumn{3}{|c}{ Coeficiente de correlação } \\
\cline { 3 - 5 } & & Imagem & Imagem & Imagem \\
& & $\mathbf{1}$ & $\mathbf{2}$ & $\mathbf{3}$ \\
\hline \multirow{3}{*}{$f \mathrm{e} \mathrm{Z} \mathrm{Z}_{0}$} & (3D/CMC) & 0,00 & 0,02 & 0,01 \\
\cline { 2 - 5 } & $(2 \mathrm{D} / \mathrm{CMC})$ & 0,02 & 0,04 & 0,04 \\
\cline { 2 - 5 } & $(2 \mathrm{D} /$ PhotoModeler $)$ & 0,01 & 0,02 & 0,02 \\
\hline \multirow{3}{*}{$\mathrm{x}_{0} \mathrm{e} \varphi$} & $(3 \mathrm{D} / \mathrm{CMC})$ & 0,00 & 0,00 & 0,00 \\
\cline { 2 - 5 } & $(2 \mathrm{D} / \mathrm{CMC})$ & 0,03 & 0,03 & 0,05 \\
\cline { 2 - 5 } & $(2 \mathrm{D} /$ PhotoModeler $)$ & 0,02 & 0,01 & 0,03 \\
\hline
\end{tabular}

Para verificar como as variações nos POI afetam os pontos determinados no espaço objeto, foram calculados separadamente a média, desvio padrão e REMQ, das coordenadas no plano dos pontos de apoio (in-plane, Tabela 8) e fora deste plano (out-of-plane-Tabela9) para os experimentos 1 e 2, que apresentaram a maior discrepância entre os coeficientes de correlação. As calibrações nos experimentos 1 e 2 foram realizadas utilizando o mesmo padrão de alvos e o mesmo programa de processamento, o que permite avaliar a influência da utilização do campo $3 \mathrm{D}$ e $2 \mathrm{D}$ nas coordenadas no plano dos pontos de apoio e fora deste plano. 
Tabela 8: Média, desvio padrão e REMQ das discrepâncias das coordenadas no plano dos pontos de apoio.

\begin{tabular}{c|c|c|c|c}
\hline \multirow{3}{*}{$\begin{array}{c}\text { Exp. 1 } \\
\text { (POI de campo 3D) }\end{array}$} & Estatísticas & $\mathbf{X}(\mathbf{m m})$ & $\mathbf{Y}(\mathbf{m m})$ & $\mathbf{Z}(\mathbf{m m})$ \\
\cline { 2 - 5 } & Media & 0,688 & $-0,540$ & 1,466 \\
\cline { 2 - 5 } & Desvio Padrão & 2,446 & 0,448 & 2,272 \\
\cline { 2 - 5 } & REMQ & 2,510 & 0,697 & 2,679 \\
\hline \multirow{2}{*}{ Exp. 2 } & Media & 0,272 & $-2,74$ & 2,184 \\
\cline { 2 - 5 } & Desvio Padrão & 2,503 & 2,452 & 3,072 \\
\cline { 2 - 5 } & REMQ & 2,441 & 3,594 & 3,673 \\
\hline
\end{tabular}

A REMQ nas coordenadas no plano dos pontos de apoio (in-plane) do primeiro experimento são inferiores a $3 \mathrm{~mm}$ (precisão média dos pontos de controle) em todas as componentes, enquanto que as coordenadas no plano dos pontos de apoio, nas componentes $\mathrm{Y}$ e $\mathrm{Z}$, no experimento 2 são superiores a $3 \mathrm{~mm}$. Observando a média constata-se a presença de tendência nessas componentes.

Tabela 9: Média, desvio padrão e REMQ das discrepâncias das coordenadas fora do plano dos pontos de apoio.

\begin{tabular}{c|c|c|c|c}
\hline \multirow{4}{*}{$\begin{array}{c}\text { Exp. 1 } \\
\text { (POI de campo 3D) }\end{array}$} & Estatísticas & $\mathbf{X}(\mathbf{m m})$ & $\mathbf{Y}(\mathbf{m m})$ & $\mathbf{Z}(\mathbf{m m})$ \\
\cline { 2 - 5 } & Media & $-1,690$ & $-2,210$ & $-3,650$ \\
\cline { 2 - 5 } & Desvio Padrão & 7,204 & 2,423 & 8,912 \\
\cline { 2 - 5 } & REMQ & 7,424 & 3,367 & 9,707 \\
\hline \multirow{2}{*}{ Exp. 2 } & Media & $-0,420$ & $-2,070$ & $-0,840$ \\
\cline { 2 - 5 } & Desvio Padrão & 7,297 & 2,336 & 10,866 \\
\cline { 2 - 5 } & REMQ & 7,308 & 3,194 & 10,900 \\
\hline
\end{tabular}

Por outro lado, a REMQ é maior nas coordenadas fora deste plano (out-of-plane) quando comparada à REMQ das coordenadas in-plane, em todas as componentes, mas a maior REMQ das discrepâncias está na componente $Z$.

Comparando os dois experimentos, a REMQ varia 1,5\% em X e 5\% em Y, enquanto em $\mathrm{Z}$ a diferença entre os campos 3D e 2D é de $12 \%$. O aumento das discrepâncias em $\mathrm{Z}$ é esperado, pois, o campo $2 \mathrm{D}$ não apresenta variação da componente $\mathrm{Z}$, o que dificulta a redução de dependência linear da coordenada $\mathrm{Z}$ do C. P. com a distância focal (Merchant, 1979; Andrade, 1981; Côrtez, 2010; Debiase, 2012).

A análise das discrepâncias das coordenadas em $\mathrm{Z}$ é interessante, pois reflete o impacto da determinação da distância focal, com o campo 3D e com o campo 2D. A diferença entre os valores da distância focal dos dois experimento é de $0,16 \%$, como apresentado na Tabela 3, sendo o valor da REMQ para a coordenada $\mathrm{Z}$, com o plano $2 \mathrm{D}, 12 \%$ maior que para o plano $3 \mathrm{D}$ em coordenadas out-of-plane.

\section{Considerações Finais}

A análise comparativa mostrou que as maiores diferenças entre os POI estimados ocorreu na distância focal. Isso ocorre porque o campo de calibração 3D permite a redução de correlação entre os valores da distância focal $(\mathrm{OI})$ e de $\mathrm{Z}_{0}(\mathrm{OE})$ de modo mais efetivo que no campo $2 \mathrm{D}$ 
(Tabela 7). A calibração com a placa 2D apresenta maiores correlações, mesmo com a aplicação do método de câmaras convergentes.

Para a análise da influência dos POI obtidos nos três experimentos de calibração, foram realizados testes simulando a existência de parâmetros de orientação exterior determinados direta e indiretamente. Com a orientação exterior direta, mesmo considerando os POE estimados simultaneamente com o conjunto de pontos tridimensionais, foi possível constatar algumas discrepâncias entre os POI estimados por diferentes configurações e programas.

Pela análise das discrepâncias apresentadas na Figura 5.a, é possível verificar que as discrepâncias apresentam padrão aleatório, o que indica que não há erros sistemáticos residuais, ou seja, os POI foram determinados com pouca correlação com os POE neste campo 3D. Nos campos 2D, por sua vez, é visível a presença de tendência em ambos os experimentos, o que indica que os POIs não foram determinados com precisão.

O cálculo das coordenadas de pontos no espaço objeto e da orientação exterior por Fototriangulação mostrou que, parte da influência da orientação interior, pode ser absorvida pela OE. Entretanto, ainda nota-se o impacto dos diferentes valores da distância focal no cálculo das coordenadas em Z. Essa diferença foi avaliada comparando-se a média, desvio padrão e REMQ das coordenadas no plano dos pontos de apoio (in-plane) e fora deste plano (out-ofplane).

Os campos 2D são amplamente utilizados pelos usuários pela facilidade em sua implantação e, principalmente, pela facilidade na identificação automática dos alvos devido à possibilidade de uso de relações de vizinhança.

Entretanto, em algumas aplicações, como em Fotogrametria Arquitetural, é importante avaliar os métodos de calibração em relação ao projeto. Para o levantamento de fachadas, por exemplo, a calibração utilizando campo 2D pode ser viável, desde que a aquisição das imagens para a calibração seja feita com distância similar à aplicada no projeto, como discutido por Brown (1971), Wang (2008) e Córdoba (2012).

Entretanto, para aplicações como a modelagem 3D de estruturas e bens arquitetônicos em geral, a calibração com o campo tridimensional possibilita parâmetros de orientação interior mais adequados.

\section{AGRADECIMENTOS}

Os Autores gostariam de agradecer a Fundação de Amparo à Pesquisa do Estado de São Paulo (FAPESP) - Processo: 2013/ 15940-9, ao Conselho Nacional de Desenvolvimento Científico e Tecnológico (CNPQ) - Processo: 130505/2013, e ao Programa de Pós Graduação em Ciências Cartográficas (PPGCC).

\section{REFERÊNCIAS BIBLIOGRÁFICAS}

Alsadik, Bashar with Fabio Remondino, Fabio Menna, Markus Gerke, and George Vosselman. "Robust extraction of image correspondences exploiting the image scene geometry and approximate camera orientation". International Archives of the Photogrammetry, Remote Sensing and Spatial Information Sciences 40 (2013): 1-7.

Andrade, José. B. Fotogrametria. Curitiba: SBEE, 1998.

Andrade, José B. and Mary Olivas A. A. "Calibração de Câmaras Aerofotogramétricas". Boletim de Ciências Geodésicas da Universidade Federal do Paraná 1, no 26 (1981): 1-39. 
Aruco: A minimal library for Augmented Reality applications based on OpenCv. Accessed in July 3, 2012 <http://www.uco.es/investiga/grupos/ava/node/26>.

Bazan, Wimerson S. with Antonio M. G. Tommaselli, Mauricio Galo and Roberto S. Ruy. "Influência das injunções de orientação relativa na calibração de um sistema dual de câmaras". Boletim de Ciências Geodésicas 15, no 3 (2009): 444-466.

Boland, Jonh. "ASPRS Camera calibration: Review painel report executive summary". Paper presented at the ASPRS Annual Conference, Portland, 2000.

Brown, Duane. C. "Decentering distortion of lenses". Photogrammetric Engineering 32 (1966): $444-462$.

Brown, Duane. C. Close-Range Camera Calibration. Photogrammetric Engineering 37 (1971): 855-866.

Brown, Duane. C. “A strategy for multi-camera on-the-job self-calibration”. Report, institute of Photogrammetry, University of Stuttgart, 1989.

Clarke, Timothy A. and John G. Fryer. "The development of camera calibration methods and models". Photogrammetric Record 16 (1998):51-66.

Conrady, A. Decentered Lens Systems, Monthly Notices of the Royal Astronomical Society 79 (1919): 384-390.

Córdoba, Paula. A. F. with Antonio A. Nepomuceno, Leonildo S. Silva, and Evangelos Christakou. "Levantamento fotogramétrico digital do palácio Itamaraty de Brasília-DF para projetos de restauração". Paper presented at the Seminário Nacional de Documentação do Patrimônio Arquitetônico com o Uso de Tecnologias Digitais, Belém, 2012.

Côrtes, João. B. R. "Análise da estabilidade geométrica de câmaras digitais de baixo custo com diferentes métodos de calibração". PhD diss., Universidade Federal do Paraná, Curitiba, Paraná, 2010.

Cramer, Michael. "Euro SDR network on Digital camera calibration". Final Report, Institute of Photogrammetry, University of Stuttgart, 2004.

Debiasi, Paula with Fabiano Hainosz and Edson A. Mitishita. "Calibração em serviço da câmara Digital de baixo custo com o uso de pontos de apoio altimétrico". Boletim de Ciências Geodésicas 18, no 2 (2012): 225-241.

Ebner, Heinrich. "Self-calibrating block adjustment". International Archives of Photogrammetry and Remote sensing 13 (1976): 128-139.

Eisenhart, Churchill. "Realistic Evaluation of the Precision and Accuracy of Instrument Calibration Systems". Journal of Research of the National Bureau of Standards C67 (1963): 161-187.

Eos Systems inc. Photomodeler: photogrammetry measurement and $3 D$ modeling software, 2013. Accessed in July, 8, 2013 < http://www.PhotoModeler.com>.

Fraser, Clive. "Automatic camera calibration in close-range photogrammetry". Paper presented at the ASPRS 2012 Annual Conference 23, Australia, 2012.

Fryer, John. G. "Camera Calibration". In: Close Range Photogrammetry and Machine Vision edited by Keith B. Atkinson, Department of Photogrammetry and Surveying, University College London: Whittles Publishing, 1996, 156-179.

Galo, Mauricio with Antonio M. G Tommaselli, Julio K. Hasegawa and Paulo O. Camargo. "Significância dos parâmetros de orientação interior na calibração de câmaras Automatic 
camera calibration in close-range photogrammetry". Paper presented at the Simpósio Brasileiro de Ciências Geodésicas e Tecnologias da Geoinformação, Recife, Pernambuco, 2008.

Habib, Ayman and Michel F. Morgan. "Automatic calibration of low-cost Digital cameras". Journal of Optical Engineering 42 (2003): 948-955.

Heikkilä, Janne. "Geometric Camera Calibration Using Circular Control Points", IEEE Transactions on Pattern Analysis and Machine Intelligence 22 (2000): 1066-1077.

Kenefick, Jonh. F. with M S. Gyer, and W F. Harp. Analytical Self-Calibration. Photogrammetric Engineering and Remote Sensing. 40 (1974).

Merchant, Dean. C. Analytical photogrammetry: theory and practice. Notes Revised from Earlier Edition Printed in 1973, The Ohio State University, Ohio State. 1979.

Merchant, Dean. C. "Aerial camera calibration: history and status". Paper presented at the ASPRS 2012 Annual Conference, Califórnia, 2012.

Mitishita, Edson and. Mary Olivas A. A. "Calibração de câmaras aerofotogramétricas a partir de aerofotos digitalizadas”. Boletim de Ciências Geodésicas 7, no 1 (2001): 64-76.

Mitishita, Edson with Paula Debiase, Fabiano Hainosk and Jorge Centeno. Calibration of low cost Digital camera using data from simultaneous Lidar and Photogrammetric survey. International Archives of the Photogrammetry, Remote Sensing and Spatial Information Sciences 39 (2012): 133-138.

Moniwa, Hideya. Analytical Camera Calibration of Close-Range Photogrammetry. PhD diss., University of New Brunswich, Canadá. 1972.

Moraes, Marcus. V. A. with Antonio, M. G. Tommaselli, Sergio L. Silva and José Marcato J. "Implantação de campo terrestre de calibração de câmaras com uso de alvos codificados". Paper presented at the VIII Colóquio Brasileiro de Ciências Geodésicas, Curitiba, Paraná, 2013.

Ruy, Roberto da S. "Desenvolvimento e validação geométrica de um sistema para mapeamento com câmaras digitais de médio formato". PhD diss., UNESP - Universidade Estadual Paulista, Presidente Prudente, 2008.

Silva, Sergio. L. A. with Antonio, M. G. Tommaselli and Almir Artero "Utilização de Alvos Codificados do tipo Aruco na Automatização do Processo de Calibração de Câmaras". Boletim de Ciências Geodésicas 20, no 3 (2014): 636-656.

Tommaselli, Antonio. M. G. and Clésio L. Tozzi. "Técnicas de calibração de câmaras em visão computacional", Paper presented at the Jornada EPUSP/IEEE em Computação Visual, São Paulo, 1990.

Telles, Sandra. S. S. and Antonio M. G. Tommaselli. "A Mathematical model for camera calibration using straight lines" Paper presented at the Euro COW 2005 International Calibration and Orientation Workshop, Castelldefels, Espanha, 2005 (CD-ROM).

Tommaselli, Antonio. M. G. with Laurent Polidori, Julio K. Hasegawa, Paulo O. Camargo, Helio Hirao, Marcus V. A. Moraes, Esrom A. Rissate Jr., Guilherme R. Henrique, Pedro A. G. Abreu, Adilson Berveglieri and José Marcato Jr. "Using vertical panoramic images to record a historic cemetery". International Archives of the Photogrammetry, Remote Sensing and Spatial Information Sciences 40 (2013): 641-646.

Trigg, Bill. Autocalibration from planar scenes. Paper presented at the europe conference of computer vision, Freiburg, 1998. 
Wang, Wenjin with Bingxuan Guo, Xin Lib and Jing Cao. "Influence factors evaluation on high-precision planar calibration of non-metric digital camera". The International Archives of the Photogrammetry, Remote Sensing and Spatial Information Sciences 37 (2008): 1071- 1076.

Wolf, Paul and Bon A. Dewitt. Elements of Photogrammetry: with Aplications in GIS. New York: McGRAW-HILL, 2000.

Zhang, Zhengyou. "A flexible new technique for camera calibration". IEEE transactions on pattern analysis and machine intelligence 22 (2000): 1330-1334.

(Recebido em dezembro de 2013. Aceito em dezembro de 2014). 